\title{
Helicopter and Aircraft detection and classification using Adaptive Beamforming and Tracking techniques
}

\author{
Ir. A.C. van Koersel \\ Dr. S.P. Beerens \\ TNO Physics and Electronics Laboratory, Group of Underwater Acoustics, \\ PO box 96864, 2509 JG The Hague, The Netherlands, \\ Tel. +31703740357, Fax +31703740651, email vanKoersel@ @el.tno.nl.
}

\begin{abstract}
Measurements of different types of aircraft are performed and used to obtain information on target characteristics and develop an algorithm to perform classification between jet aircraft, propeller aircraft and helicopters. To obtain a larger detection range, reduce background noise and to reduce classification errors in a multi-target environment, a real time adaptive beamformer algorithm is developed for a three microphone array. The output of the beamformer is submitted to a tracking algorithm. Acoustic signals from identified tracks are submitted to the classification algorithms. The algorithm is tested on data recorded during various field trials. The objective of the research, which is part of a research program for the Dutch Army, is to detect the passage of an aircraft with one or more mechanical wave sensors, either acoustic or seismic. After detection of a target, classification of the type of aircraft is requested (for example: helicopter-jet-propeller-rpv). If possible type identification is also requested. Earlier work showed promising results for detection and classification of helicopter targets. The projects resulted in an algorithm that can detect and classify helicopters, but it was developed to reject other targets. The chosen approach is to combine new aircraft detection and beamforming algorithms with the existing algorithms.
\end{abstract}

Keywords: acoustic, detection, classification, helicopter, aircraft, adaptive beamforming, tracking.

\section{BACKGROUND AND INTRODUCTION}

The military interest in acoustic-seismic aircraft detection is based on the military requirement to obtain situational awareness on the battlefield. Traditionally the intelligence about aircraft movement is gathered with (radar) or electro-optic sensors. However there are possibilities that such assets are not available or do not function as expected due to lack of coverage or line of sight conditions. An alternative method for detection of targets, less hampered by line of sight requirements is the use of Unattended Ground Sensors (UGS), for example using seismic or acoustic transducers. More information on the military perspective of the use of UGS is given in [Stotts 1]. An algorithm developed at TNO-FEL can be used to detect and classify helicopter targets, using the acoustic signal from a microphone or geophone as input. Originally a laboratory version implemented in Matlab of a helicopter detection demonstrator was developed based on a desktop PC [van Koersel 2,3]. Later the demonstrator was extended to detect passing aircraft, and to automatically discriminate between passes of jet or propeller aircraft. The work regarding aircraft detection and classification is described in detail in [van Koersel 4,5]. It is noted that relatively successful results are reported on the detection and classification of the type of aircraft (jet or propeller) passing over a sensor array. The investigation of the possibilities of the identification of the aircraft model shows less promising results, broadband signatures of jet aircraft show comparable difference between passes of aircraft of the same model, as they do between passes of different aircraft. It seems that engine power settings and the flight profile are important factors that determine the spectral properties of the aircraft passage signatures. The current paper describes the implementation and application of a Delay and Sum (DS) beamformer and an adaptive Minimum-Variance Distortionless Response (MVDR) beamformer to data recorded using a triangular microphone array. A comparison is shown between results of both beamforming methods. The adaptive beamformer is combined with a clutter removal and tracking algorithm developed for underwater applications. Identified tracks can be submitted to the existing helicopter detection and classification algorithm. Results using this combination are compared to results obtained using single sensor input, using real data. 


\section{BEAMFORMER DESCRIPTION}

Beamforming techniques to obtain target localisation are described in different papers, for example in the proceedings of last years Spie Aerosense conference session on UGS [Carapezza 6]. Arrays used for the estimation of the target bearing range from 8 sensors in a wagon-wheel arrangement [Kaplan 7], a similar arrangement using 7 sensors [Kozick 8] and circular arrays using 8 microphones [Johnson 9]. Beamforming techniques are also used extensively for underwater applications, to determine target bearings, to reduce the influence of background noise and to increase detection ranges. Traditional techniques applied to towed arrays are based on conventional delay and sum beamforming techniques. Improvements to obtain port-starboard discrimination of targets has led to the use of twin arrays, and later triplet arrays. A triplet array is an array of hydrophones arranged in a triangle in a single array tube. The array output is beamformed, subsequently the beamformed result is treated as a triangle of directional hydrophones and submitted to the triplet beamformer. In [Beerens 10] different beamforming techniques for hydrophone triplet arrays are described and applied to measured data. We have applied the triplet array beamforming method to the triangular microphone array (1.5 $\mathrm{m} \mathrm{spacing)}$ used by TNO-FEL for measuring acoustics targets [van Hoof 11].

\subsection{Delay and Sum Beamformer}

The conventional delay and sum beamformer is formulated as follows. First the data is transformed to the frequency domain (by means of an FFT): $\boldsymbol{x}_{k}(t) \Rightarrow \boldsymbol{X}_{\boldsymbol{k}}(f)$, where $k=1,2,3$ numbers the microphones. In the frequency domain a time delay corresponds to a phase shift and the 3 microphone outputs can be summed with a compensating phase shift:

$$
p(\theta)=\sum_{k} \boldsymbol{X}_{\mathrm{k}}(f) \exp \left(-2 \pi \mathrm{i} f \tau_{k}\right),
$$

where the delay times $\tau_{k}$ depend on the direction of arrival of arrival (bearing) denoted by $\theta$ as :

$$
\begin{aligned}
& \tau_{1}=-d / c(1 / 3 \sqrt{3} \cos \theta) \\
& \tau_{2}=+d / c(1 / 6 \sqrt{3} \cos \theta+1 / 2 \sin \theta) \\
& \tau_{3}=+d / c(1 / 6 \sqrt{ } 3 \cos \theta-1 / 2 \sin \theta)
\end{aligned}
$$

The microphone array is an equilateral triangle with sides $d, c$ is the speed of sound. For all bearings the three outputs are combined in a different way, but only for the true target bearing the three outputs are summed coherently (in phase). Only for this bearing the beamformer output will be 3 times higher than a single microphone output, resulting in a maximum processing gain of $5 \mathrm{~dB}$. More important than this extra gain may be the ability of the beamformer to estimate the bearing of the target. Although with only three microphones in the array the beamwidth is rather high, the bearing can be estimated quite accurately in case of high SNR.

\subsection{MVDR beamformer}

In the Minimum-Variance Distortionless Response (MVDR) beamformer one maximises the beam output $S N R$ by means of minimising the total output power while maintaining a constant gain in a specified beam direction. The goal is to suppress directional noise from sources outside the specified beam by adaptively changing the array beamformer, so that the sidelobes of these sources are lowered under the background noise.

An additional advantage of this process is a reduction of the beamwidth. In the mathematical description we will use a matrix notation to reduce the writing. The (unweighted) conventional DS beamformer in this notation is (cf. Eq. (1)):

$$
p=\mathbf{d}^{*} \cdot \mathbf{X}
$$

and the power is

$$
p^{2}=\left|\mathbf{d}^{*} \cdot \mathbf{X}\right|^{2}=\left(\mathbf{d}^{*} \cdot \mathbf{X}\right)\left(\mathbf{X} \cdot \mathbf{d}^{*}\right)^{*}
$$

where $\mathbf{d}$ is the steering vector and $\mathbf{X}$ is the vector with complex microphone data for one frequency bin, both have a length $K=3$.

In the adaptive (MVDR) beamformer the steering vector is replaced by an optimal steering vector [Nielsen 12]: 


$$
p=\mathbf{w}^{*} \cdot \mathbf{X}
$$

where the optimal steering vector is related to the covariance (or correlation) matrix $\mathbf{R}=\mathbf{X X}^{*}$ :

$$
\mathbf{w}=\mathbf{d} \mathbf{R}^{-1} /\left(\mathbf{d}^{*} \mathbf{R}^{-1} \mathbf{d}\right)
$$

In the case of uncorrelated noise the correlation matrix has only contributions from the auto-correlation's on the diagonal, i.e. $\mathbf{R}=\mathbf{I}$. In this case the adaptive beamformer is equivalent to a conventional beamformer. For data with directional noise the matrix departs from unity and the adaptive beamformer becomes efficient.

The MVDR beamformer output power now becomes:

$$
p^{2}=\mathbf{w}^{*} \mathbf{R} \mathbf{w}=\mathbf{d}^{*} /\left(\mathbf{d}^{*} \mathbf{R}^{-1} \mathbf{d}\right) \mathbf{R} \mathbf{d} /\left(\mathbf{d}^{*} \mathbf{R}^{-1} \mathbf{d}\right)=\mathbf{d}^{*} \mathbf{R} \mathbf{d} /\left(\mathbf{d}^{*} \mathbf{R}^{-1} \mathbf{d}\right)^{2}=\left(\mathbf{d}^{*} \mathbf{R}^{-1} \mathbf{d}\right)^{-1}
$$

The R's and d's have been eating each other and we are left with a relatively simple expression in which the inverse covariance matrix $\mathbf{R}^{-1}$ plays the central role. It is sandwiched between two conventional steering vectors. In spite of the simplicity of the MVDR algorithm there are some problems with the implementation of this beamformer. These problems are:

- How to compute $\mathbf{R}$ ?

- Can we invert $\mathbf{R}$ ?

The matrix $\mathbf{R}$ depends on time and frequency. It is hard to determine the proper integration time and bandwidth to compute $\mathbf{R}$ in a robust way, without smearing out vital information. Integration times should be short to cope with rapid varying noise sources, but long enough to obtain statistically stable information. The tuning is complicated (it depends on the sensor, the environment, the self-noise, etc).

The inversion of $\mathbf{R}$ is sometimes cumbersome. Since $\mathbf{R}$ is estimated from measurements, with associated measurement errors, some matrix elements may be poorly estimated. Especially in the cases of interest (with high non-diagonal elements) the matrix may become nearly singular. The condition number (smallest eigenvalue over the largest eigenvalue) becomes close to machine precision and inversion gets unstable. A trick to get over this problem is diagonal loading. By addition of small values to the auto-correlation the invertability is restored. Of course this non-physical addition decreases the performance. In fact the MVDR beamformer is mixed with a DS beamformer and if large loading is required the performance will be no better than that of a DS beamformer. To find a stable but low loading requires again a lot of tuning.

\subsection{Application to a three-microphone array}

The main problem in practical application of MVDR to sonar arrays is to find a reliable estimate of the covariance matrices. Problems arise from the fact that loud nearby noise sources do not "stay in the same beam" long enough to provide an accurate, time-stationary estimate of the matrix elements. Still an attempt was made.

The covariance matrices $(\mathbf{R})$ are computed in the frequency domain. The integration time is $1 \mathrm{~s}$ and the integration bandwidth is $3 \mathrm{~Hz}$. The results are very sensitive to these parameters. Longer integration gives more robust results, but the averaging also fades out the instantaneous noise bursts to which one actually wants to adapt to.

The inversion of $\mathbf{R}$ takes place after scaling the matrix (by $3 /$ trace $(\mathbf{R})$ ), such that the average diagonal element is 1 . A small loading $\left(10^{-2}\right)$ was found large enough for stable condition numbers. 


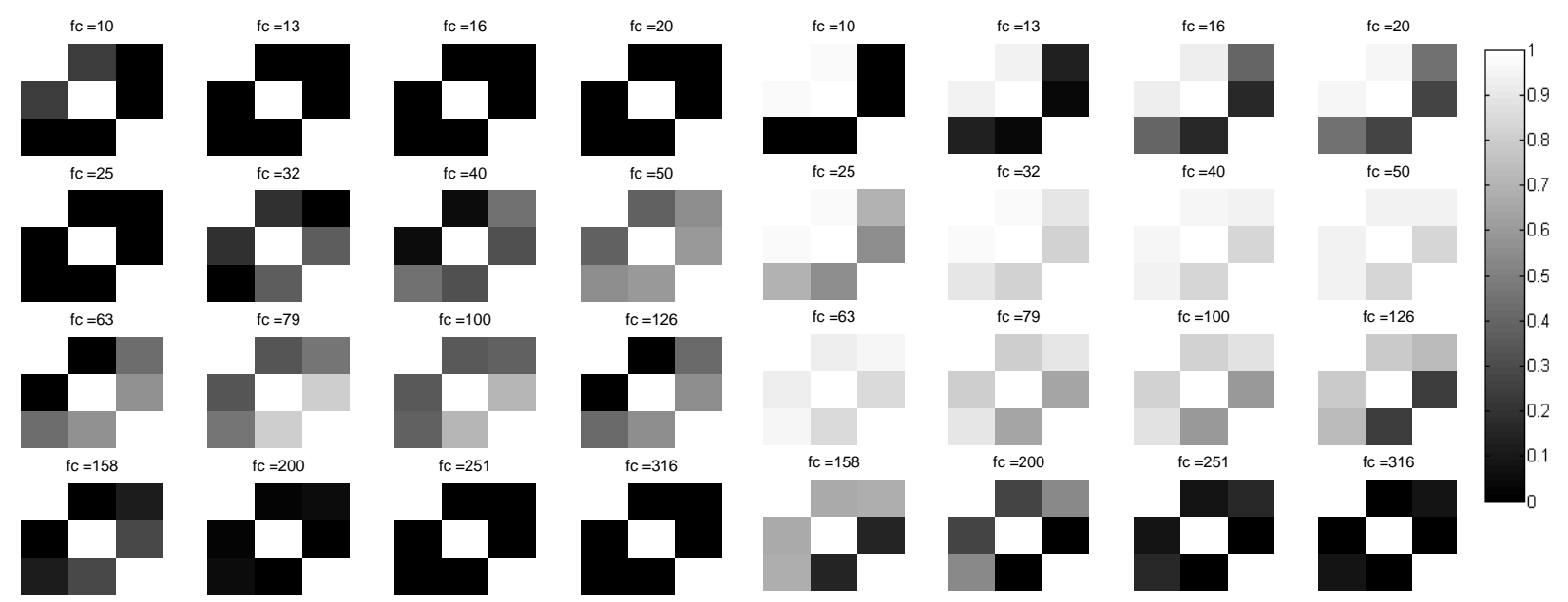

Figure 2.1: $\quad$ Scaled covariance matrices for only rain and wind noise (left) and for wind and helicopter noise (right)

In Figure 2.1 16 typical examples of $\mathrm{R}$ are plotted using experimental data. The matrices are averaged in terts band frequencies, starting at $f c=10 \mathrm{~Hz}$. In the case that only onmi-directional noise (wind and rain) is received (left-hand panel), the matrices look very much like the identity matrix.

The three microphones show no correlation for wind noise. All the off diagonal components are close to 0 . In that case MVDR beamformers are mathematically equivalent to conventional beamformers. However, in case of directional noise bursts (e.g. from the passing helicopters) off-diagonal elements become alive, in this figure mostly for the lower frequency bands (for this type of noise the spatial correlation function is $\cos (k x)$ ). Here the MVDR beamformer become effective.

To apply the MVDR beamformer algorithm to our three-microphone array, we have derived the analytical expression for the inverse of the covariance matrix. Given the input from the three sensors we calculate $\mathbf{R}$ without the use of matrix inversion routines, which increases accuracy and decreases computation time.

\section{COMPARISON OF DS AND MVDR BEAMPATTERNS}

To compare the results of both beamforming methods, data gathered during a field trial is processed, and beampatterns are calculated using both the DS and the MVDR beamformer. For both methods 72 beams covering the whole horizon were calculated. The data originates from a field trial held in May 1999 on Soesterberg airbase in the Netherlands. A map of the measurement location is shown in figure 3.1. 


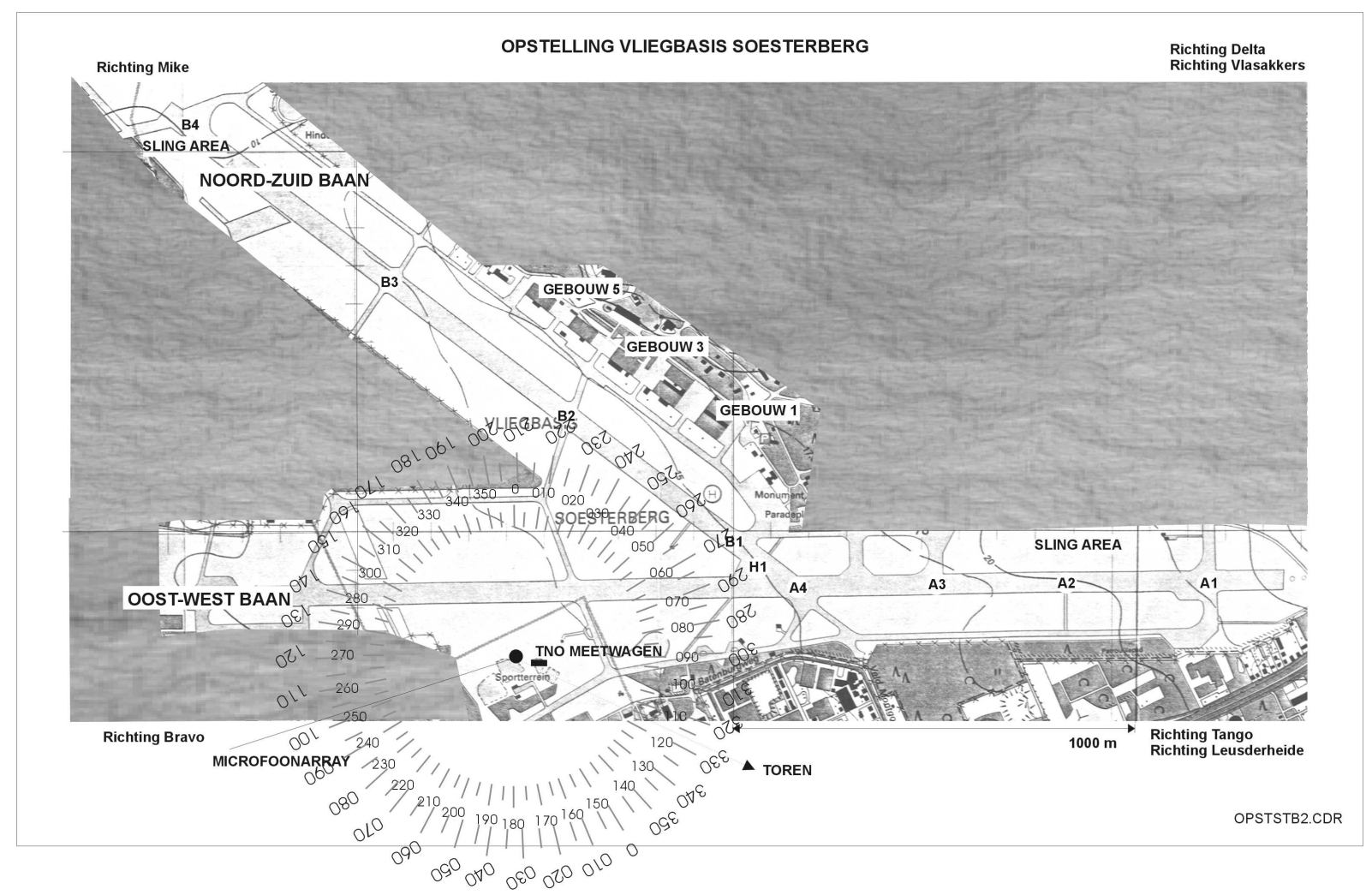

Figure 3.1: Measurement position on Soesterberg airfield. The microphone array is located in the middle of the compass ring, the outer ring shows the direction of the microphone array, with M1 direction (0) pointing towards 150 degrees compass direction.

The microphone array was located in the centre of the two compass circles. The inner circle shows the compass headings ( 0 upwards), the outer circle shows the heading of the microphone array, with microphone 1 pointing towards compass direction 150. Table 3.1 gives some information on the type of aircraft present in the recording used for comparison of the beampatterns. The recording duration is approximately 8 minutes, it contains approaches of 2 helicopters (both Cougar), and one transport aircraft (Fokker 27).

Table 3.1 Recording info of run 6_9 of May 27, 1999 at Soesterberg airbase.

\begin{tabular}{|l|l|l|}
\hline $12: 02.20$ & RR & Start recording \\
\hline $12: 02$ & Cougar 1 & $\begin{array}{l}\text { From 270 over East-West runway to intersection in front of } \\
\text { measurement van }\end{array}$ \\
\hline $12: 03$ & Cougar 1 & Lands in front of building 3, taxi to building 1 \\
\hline $12: 05$ & Cougar 2 & Lands in front of building 1 \\
\hline $12: 06$ & F27 & Lands on East-West runway from direction 270 \\
\hline $12: 10.51$ & SR & Stop recording \\
\hline
\end{tabular}

The compass bearing for microphone 1 was approximately $150^{\circ}$, which means that the aircraft arriving from bearing 270 are seen by the array in a direction of approximately $120^{\circ}-130^{\circ}$ degrees. The bearing towards the buildings mentioned in table 1 are approximately 230-260 degrees. 

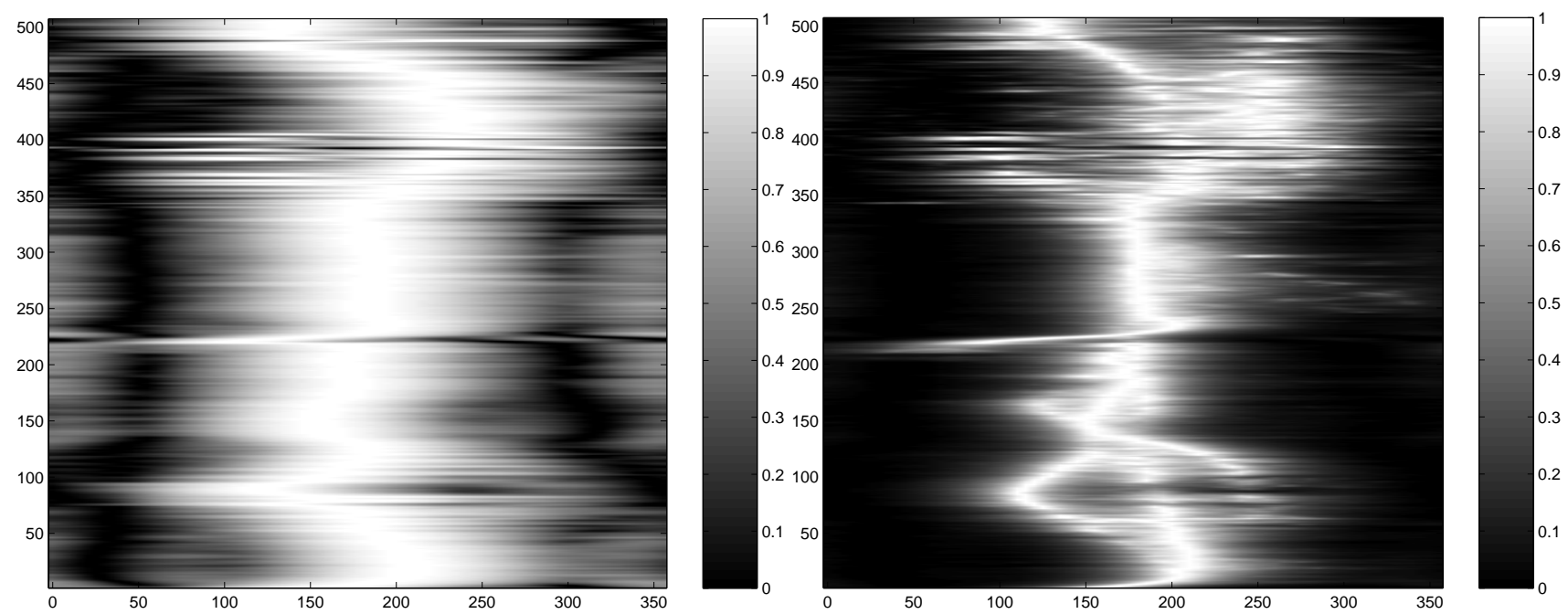

Figure 3.2 Beampatterns calculated for both DS (left) and MVDR (right) beamforming algorithms using run 6_9 recorded at Soesterberg airfield. The bearing is shown at the $x$ axis in degrees, the recording time is shown along the y axis. Both beampatterns are scaled between 0 and 1.

Inspecting both beampatterns, the most striking difference is the beamwidth and the associated possibility to separate different sources. In the right graph we are able to separate three sources (between $t=50 \mathrm{~s}$ and $150 \mathrm{~s}$ ), using the DS beampattern that is not be possible.

The beampattern resulting from the calculation of the MVDR beamformer is used as input for an algorithm to reduce the detection of false targets. First local maxima are identified in the beampattern without using a threshold, so that low SNR targets are not lost. Clutter removal is performed by the convolution of a detection window and the local maxima. An example of the target detections extracted from the MVDR beampattern show in figure 2.3. The left graph shows the target detections after clutter removal, the right graph shows the original MVDR beampattern.
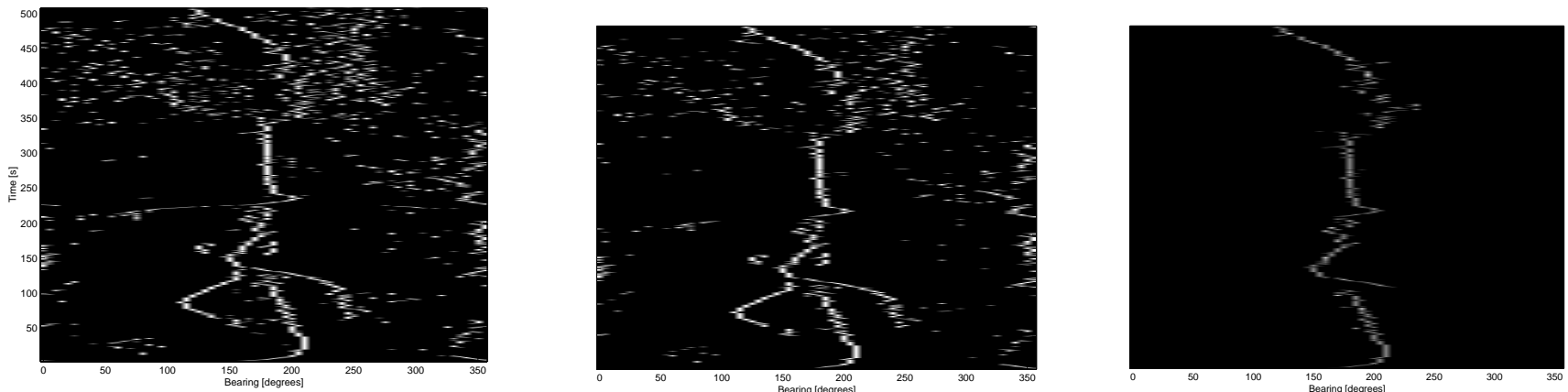

Figure 3.3 Left: Local maxima in the MVDR beampattern, Middle: target detections after clutter removal, Right: tracked beam.

After beamforming, clutter removal and tracking, the tracked beam can be submitted to the classification algorithm, to determine the type of target present in the beam. To facilitate comparison between single sensor results and the results after beamforming, we have chosen to use the algorithm used to determine the type of helicopter. The algorithm was described earlier in [van Koersel 2]. 


\section{CLASSIFICATION RESULTS AFTER BEAMFORMING}

To compare results of the helicopter classification algorithm on both beamformed and single sensor data, we present the time-frequency distribution of both the single sensor and the beamfomed data, as well as the classification results obtained using the data as input for the classification algorithm. For the single sensor data this is straightforward, the pre-processed sensor data can be submitted to the algorithm immediately. To be able to show classification results after beamforming, we have to identify a target track first. After track identification, we calculate one beam to the target, for the entire frequency range of interest. The tracked beam is submitted to the classification algorithm. In our current implementation the target track has to be identified manually at first, an algorithm performs automatic track following afterwards. The example shown here is the approach of a Lynx helicopter from a range of approximately $8 \mathrm{~km}$, recorded during measurements in Dreux France, 1988 [vanHoof 11]. Close inspection of the time frequency plot of the single sensor data (figure 4.1 left) shows that this dataset is extremely difficult. It contains amplifier switching, necessary since the data was recorded using a tape recorder with a dynamic range not exceeding $50 \mathrm{~dB}$, as well as data that is nearly at the noise floor of the AD converter used.
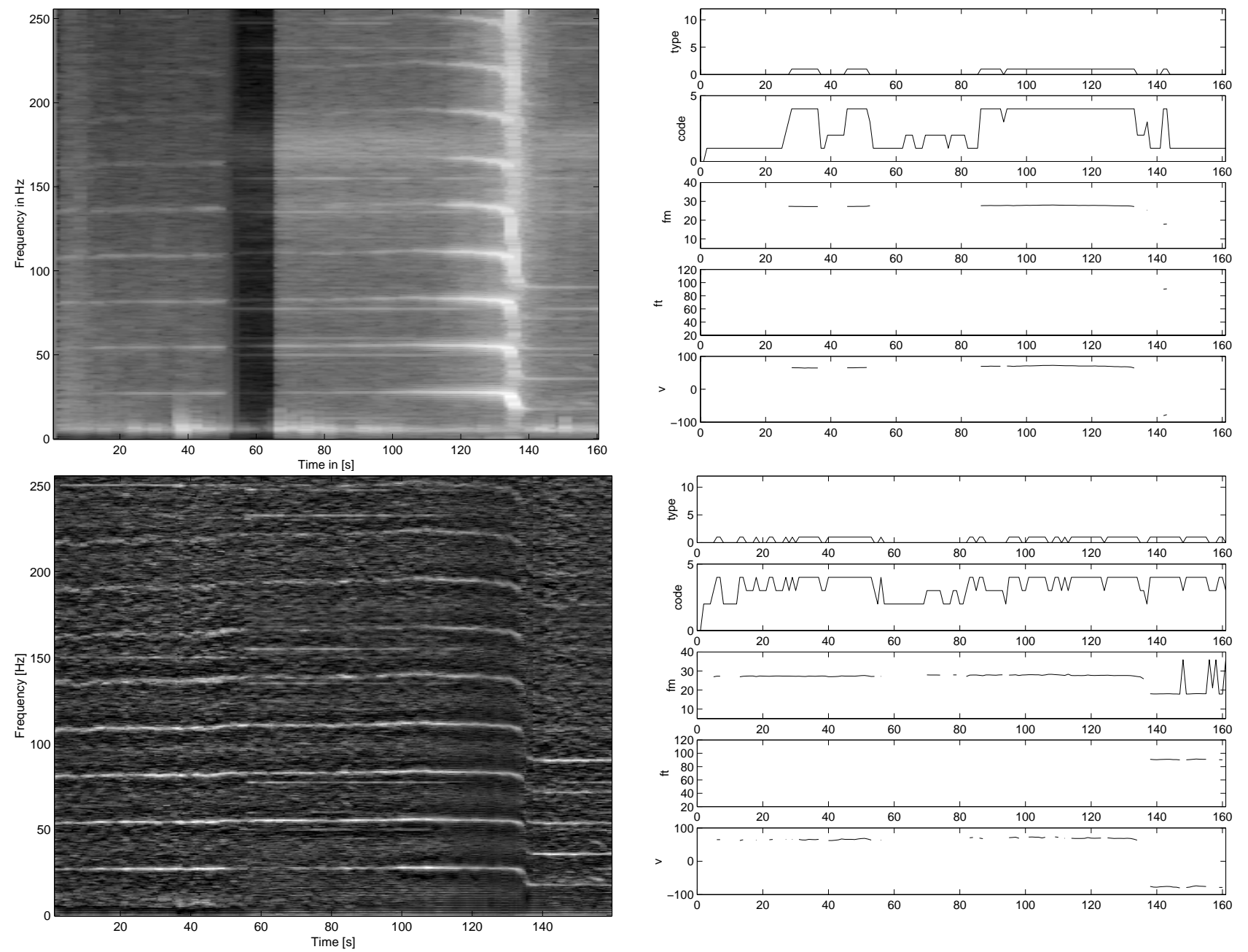

Figure 4.1 Results of the helicopter classification algorithm for a single sensor(top graphs) and after beamforming and tracking (bottom graphs. One helicopter is approaching the array from a distance from approximately $8 \mathrm{~km}$. The left graph shows the time frequency distribution, the right graph shows the output derived from the classification algorithm (from top to bottom: helicopter type, classification code, main rotor frequency, tail rotor frequency and speed relative to the sensor). 
Comparing the graphs with the classification results show that the classifier connected to the MVDR beamformer can pick the helicopter up $4 \mathrm{~s}$ after the start of the recording, where as the single sensor classifier picks the helicopter up after $27 \mathrm{~s}$. With a helicopter approach speed of $65 \mathrm{~m} / \mathrm{s}$ in this run that means a difference in detection range of approximately $1500 \mathrm{~m}$. The algorithm connected to the MVDR beamformer classifies 55\% of the samples correctly, the single sensor classifier scores $40 \%$ correct. Note that in both cases for this dataset there are no samples that are classified the wrong type.

Figure 4.2 show the results on a single sensor as presented in [van Koersel 2], the dataset was recorded at Soesterberg 1999. Two Cougar helicopters are approaching and landing after eachother, one stationary CH 47 starts up on a platform near its hangar. For this paper we have processed the first part of the recording, with the two helicopters approaching and the stationary one starting up.
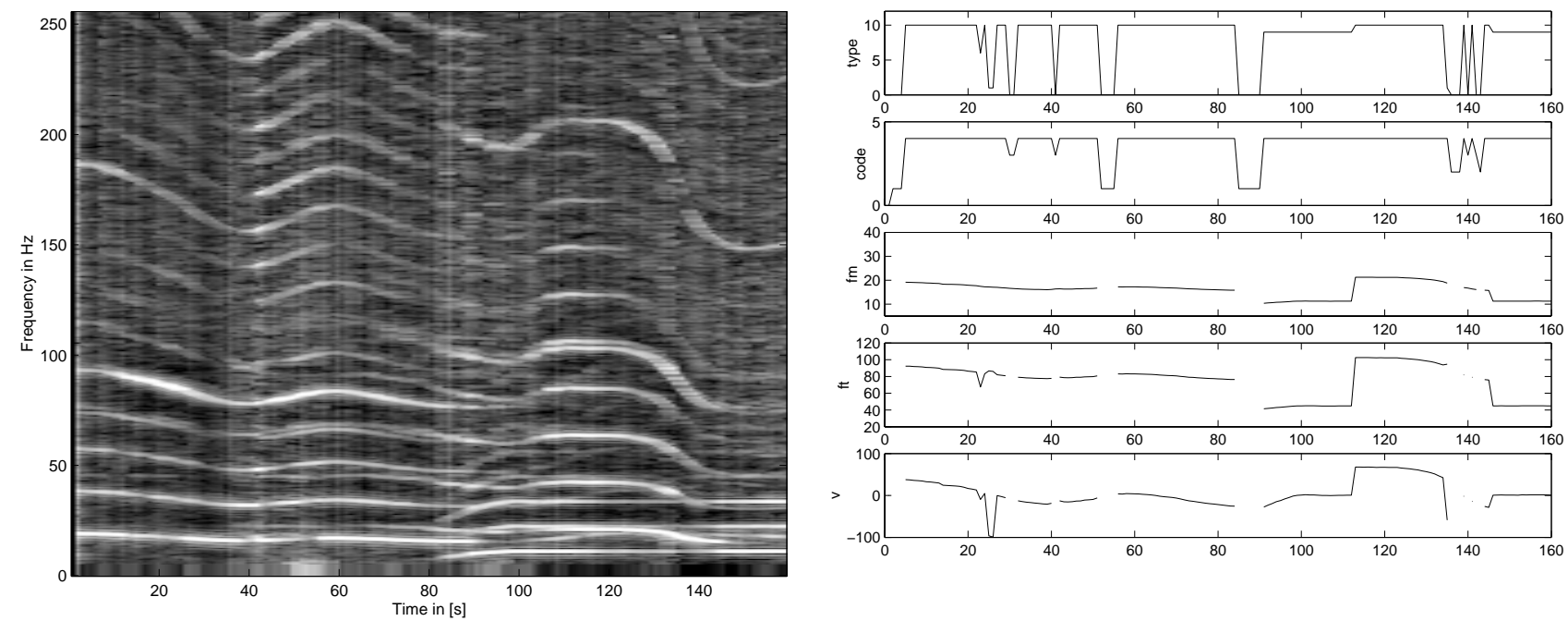

Figure 4.2: Time frequency distribution of multiple helicopters, two of type 10, one of type 9. Horizontal: time axis (0160s), Vertical: frequency axis. Right: Classification results on the data, from top to bottom: helicopter type, classification code, main and tail rotor frequency and helicopter speed.

To show the ability of the MVDR beamformer to separate targets, we have processed the first part of the recording using the MVDR beamformer and tracker. Figure 4.3 shows only the beams that were tracked, the data from the tracked beams was submitted to the classification algortihm.

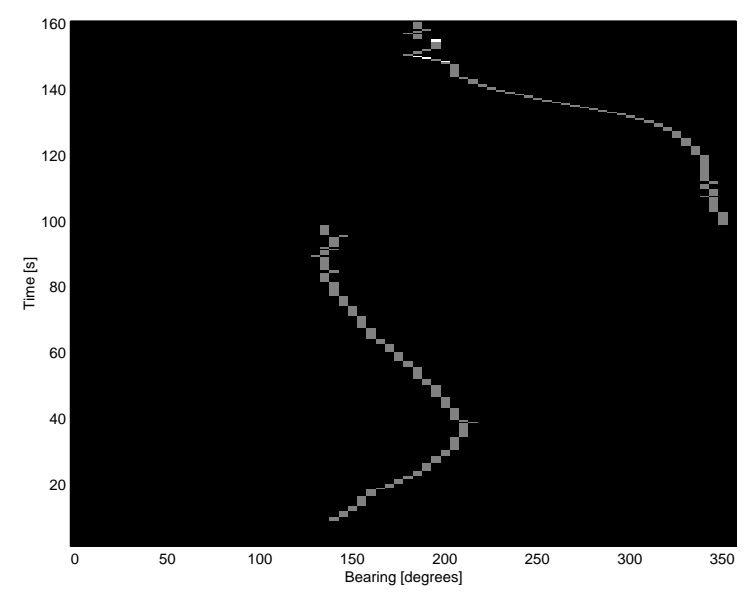

Figure 4.3: $\quad$ Tracked beams of the recording with multiple helicopters. 

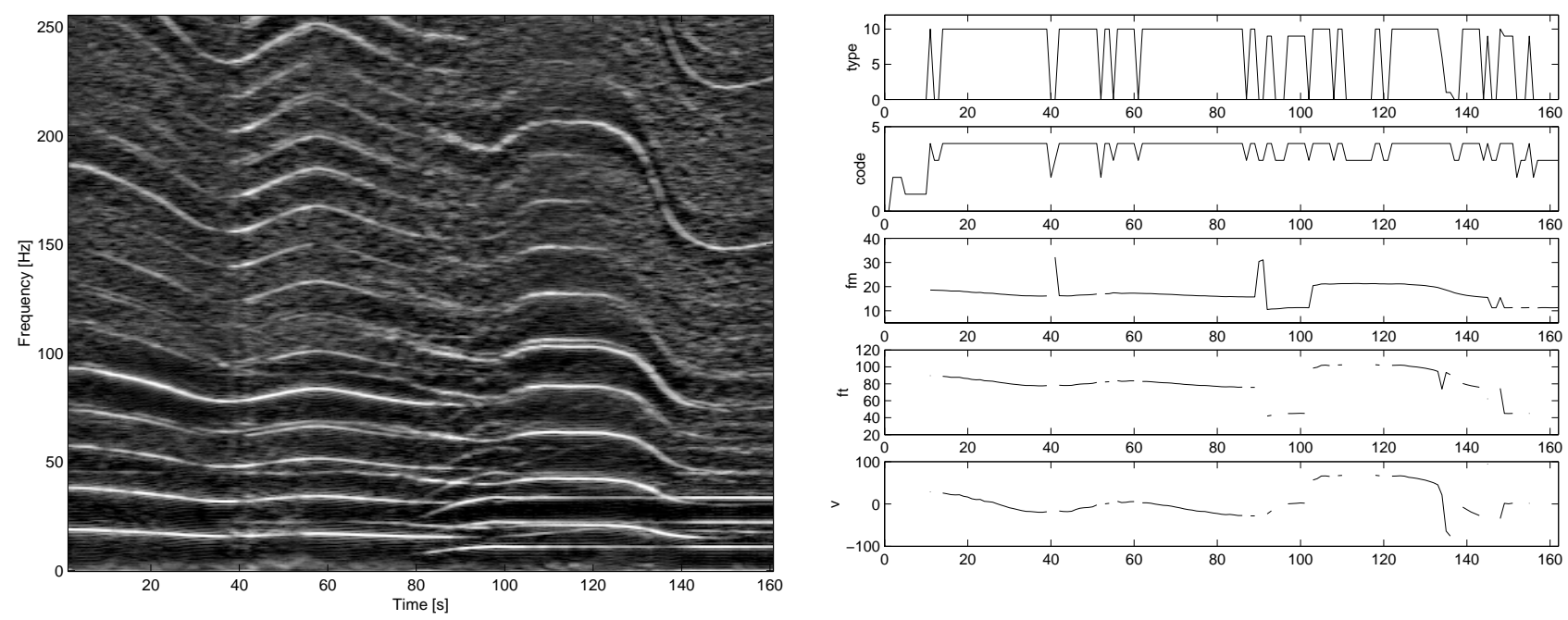

Figure 4.4: Time frequency distribution of multiple helicopters, two of type 10, one of type 9, Horizontal: time axis (0160s), Vertical: frequency axis. Right: Classification results on the data, from top to bottom: helicopter type, classification code, main and tail rotor frequency and helicopter speed.

The first helicopter that is approaching is correctly classified as type 10, until the bearing is close to the bearing of the stationary helicopter, then the classifier switches to the louder stationary helicopter. As soon as the new arriving helicopter appears in the beampattern, the tracker is pointed to that one. The classifier now switches correctly to type 10. The louder stationary helicopter is surpressed, until the bearing of the arriving helicopter is within 20-30 degrees of the bearing of the stationary one (after $\mathrm{t}-140 \mathrm{~s}$ ). There are some problems with correct classification, the main rotor is picked up correctly however.

\section{CONCLUSIONS}

MVDR applied to a triangular microphone array allows the separation of targets that are not identifiable as separate targets using conventional beamfomers. The drawback of MVDR is usually loss in frequency resolution, the chosen implementation with averaging over only three frequency bins results in an output spectrum with enough resolution to be submitted to algorithms that are used for type classification. Calculation on stored datafiles is faster than real time on a 800 $\mathrm{MHz}$ Pentium PC running Matlab. The overall result on classification performance is a larger detection range and less false classifications in the presence of interfering targets.

\section{ACKNOWLEDGEMENTS}

The research is partly funded by the Royal Dutch Army, the project officer resides at the School of Military Intelligence. The field trails were performed with the enthusiastic support of the personnel of the airfields and the RNLAF airshow organisation committee.

\section{REFERENCES}

1. L.B. Stotts, Unattended ground-sensor-related technologies: an Army perspective, in Edward M. Carapezza, Todd M. Hintz, Unattended Ground Sensor Technologies and Applications II, Proceedings of Spie Aerosense 2000, Orlando Florida.

2. A.C. van Koersel, Helicopter detection and classification demonstrator, In Unattended Ground Sensor Technologies and Applications II, Edward M. Carapezza, Todd M. Hintz, editors, Proceedings of SPIE Vol. 4040 (2000), pp. 121-130.

3. A.C. van Koersel, Acoustic Helicopter Classification Technology Demonstrator, FEL-00-A083. 
4. A.C. van Koersel, Acoustic Helicopter and FW Aircraft Detection and Classification, pp. 108-118 of the Proceedings of the Ninth Annual International Symposium on Long-Range Sound Propagation, Held at TNO-FEL, The Hague, Published by the University of Missisipi, NCPA Report Number DM0501-01.

5. A.C. van Koersel, Aircraft Detection and Classification using Acoustic Sensors, FEL-01-A145.

6. Carapezza, Unattended Ground Sensor Technologies and Applications III, Spie vol 4393, April 2001.

7. L.M. Kaplan, P. Molnár, Q. Le, Bearings-only target localization for an acoustical unattended ground sensor network, Spie vol 4393, pp. 40-51.

8. R.J. Kozick, B.M. Sadler, Near-field localization of acoustic sources with imperfect spatial coherence, distributed processing, and low communication bandwidth, Spie vol 4393, pp. 52-63.

9. K.B. Johnson, R.L. Wright, J. Barnes, Distrbuted acoustic beamforming using COTS technology and Microsoft Windows CE 3.0, Spie vol 4393, pp. 64-68.

10. S.P. Beerens, R. Been, J. Groen, Y. Doisy \& E. Noutary, Adaptive port-starboard beamforming of triplet arrays, Proceedings of UDT Pacific, 2000.

11. H.A. van Hoof, A.I. Bol, collection of helicopter signatures with mechanical wave sensors, phase 2, FEL-90-A156, aug 1990.

12. R.O. Nielsen, Sonar Signal Processing, Artech House, 1991. 